\title{
Analytical Modelling and Implementation of a New Four-Switch Hybrid Power Filter Topology
}

\author{
Jiri Klima \\ Department of Electrical Engineering and Automation \\ Technical Faculty of CZU in Prague \\ 16627 Prague 6, Czech Republic \\ E-mail: klima@tt.czu.cz
}

\begin{abstract}
This paper presents a new three-phase four-switch shunt hybrid power filter with the new circuit topology aimed at compensating reactive power and higher harmonics of the load. A space-vector PWM scheme is adopted to reduce the switching state of power switches. In the proposed control scheme, proper voltage vectors are generated on the ac terminal for drawing nearly sinusoidal line currents with controllable power factor. An original closed-form solution of line currents, based on the mixed $p-z$ approach is presented. The analytical procedure is verified by experimental results to confirm the effectiveness of the proposed control scheme.
\end{abstract}

\section{Key words}

Hybrid power filter, harmonic and reactive power compensation, reduced circuit topology, close-form analytical solution

\section{Introduction}

In recent years, harmonics pollutions have become a serious problem in power distribution system. Current and voltage harmonics have negative effects on the operation of the electric power system. Thus, great attention is focused on harmonic generation and control. Several standards have introduced limits on current harmonics injected into the power system and on voltage harmonics at the bus-bars of the system.

A conventional measure for the compensation of nonsinusoidal currents and/or lagging power factor of a load, is represented by tuned passive filters (PF) connected in parallel to the load. However, their frequency characteristics are unfavorably influenced by a variable grid impedance and here, in reality, the danger of the excitation of resonance exists. However, the variations of the filter parameters occur due to aging, temperature, and other operations conditions.

The active power filter (APF) made up of a power converter has been developed to suppress the harmonic currents and improve powder factor. The APF may
Jiri Skramlik, Viktor Valouch

Institute of Thermomechanics

Academy of Sciences of the Czech Republic

Dolejškova 5, 18202 Praha 8, Czech Republic

E-mail: valouch@iee.cas.cz

substitute for passive ones in some cases, or serve as an additional tool, together with $\mathrm{PF}$, for improving the harmonic spectra of the load current and supply voltage. The active filters may be also used as voltage stabilizers at the point of coupling or as power flow controllers. Contrary to PF, the function of active filters is influenced substantially neither by ageing nor by changes in the grid impedance. But the main disadvantage for the use APF in large scale is that complex control and cost of power converter become very high thus limiting their applications.

Hybrid power filters (HPF) consisting of dedicated passive and active filters connected in series or parallel has been published in the literature. The tolerances and variations in the hybrid filter are not as serious as those in a passive filter used alone. It is also well known that HPF helps to reduce the active filter rating.

Some research efforts have been made to develop new power converters with reduced losses and costs .Among these circuits, the three-phase converters with only two inverter legs (B4), is an alternative solution. Only four power switches and four clamping diodes are used in the adopted converter instead of six power switches and six clamping diodes used in a conventional converter. With a reduced number of power switches the converter can compensate reactive power and unbalanced harmonics also in case of unbalanced source voltages. It is possible to obtain near-unity power factor and input currents with nearly sinusoidal shape, strongly reducing line current harmonics.

But, the main disadvantages of this topology are as follows:

i) The center of the dc-bus voltage must be accessible, as one of the three converter phases is directly connected with this center point.

ii) The size of the dc-link capacitor must be high to prevent a pulsation of the dc-bus center point as the current going through this point is not dc but it has ac character. 
As for modelling of PWM rectifiers and APFs, some professional SW tools (e.g. PSpice, Matlab-Simulink) are extensively used in power electronic circuits. However, some difficulties in these professional environments occur from time to time by solving either steady states or transient processes connected with PWM systems, namely long execution time, numerical convergence problems, etc.

Thus, a promising solution seems to be an exact analytical one, especially from the viewpoint of obtaining performance equations from which the rms and average values of voltages and currents may be obtained directly, without the need to perform numerical treatment of the system. However, an analytical closed-form solution for high frequency PWM systems is difficult to find, due to many algebraic equations that need to be solved. The second very popular approach, solution in the frequency domain, also requires enormous mathematical efforts for the system in question.

So, we used a very new method, mixed p-z approach [4], to analyze the suggested modification of the SVM for the component minimized PWM rectifiers. First, unsymmetrical voltage space-vectors are decomposed into symmetrical components using Discrete Fourier Transform (DFT) then, for the voltage symmetrical components; the mathematical model makes use of the Laplace and modified Z-transform to predict response of the line currents. From the Laplace transform of space vectors we can also derive the Fourier spectrum. In this paper, a novel circuit topology shown in Fig.1 is proposed and investigated.

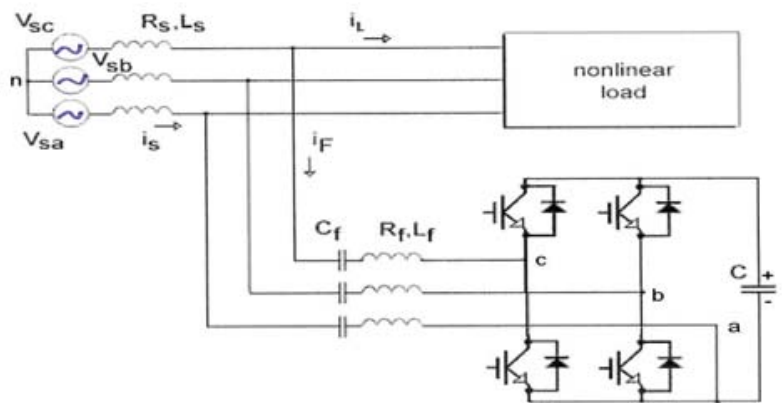

Fig.1 Configuration of the proposed topology

As can be seen in this configuration one phase (in our example phase "a") of the passive filter is directly connected with any one of the de terminal of a fourswitch converter (in our example minus dc bus terminal). Consequently, the number of power switching devices is reduced and disadvantages i)-ii) is avoided, as dc-mid bus point is not utilized.

\section{System configuration and analysis}

The system configuration is shown in Fig.1.The passive part of the filter consists of series resistor, inductance and capacitor $\mathrm{R}_{\mathrm{f}}, \mathrm{L}_{\mathrm{f}}$, and $\mathrm{C}_{\mathrm{f}}$, respectively. The voltage-source power converter with only two arms is controlled by the space-vector pulsewidth modulation (SVPW) strategy. The SVPWM strategy is described in [7].

The situation is shown in Fig.2.We can see the voltage space-vectors in normal VSC with sixth switching devices (B6 connection).In that topology we have at disposal 6 non-zero vectors $\left(\mathbf{V}_{\mathbf{1}}-\mathbf{V}_{\mathbf{6}}\right)$ and one zero vector $\mathbf{V}_{\mathbf{0}}$. As in our new topology the phase $\mathrm{a}$ is connected with negative dc bus terminal we can use only 4 vectors shown in the shade area $\left(\mathbf{V}_{\mathbf{0}}, \mathbf{V}_{\mathbf{3}}, \mathbf{V}_{\mathbf{4}}\right.$ and $\mathbf{V}_{\mathbf{5}}$ ). But as every phase of the proposed HPF contains a capacitor, which can block the dc-component of the power converter's output, we can see that this spacevector diagram contains a dc offset voltage in every of three phases.

It means that from the ac utility point of view the voltage space vector diagram in Fig. 2 is shifted by a dc-offset voltage space-vector

$$
\boldsymbol{V}_{\mathrm{off}}=-V_{\mathrm{dc}} / 3 \text {. }
$$

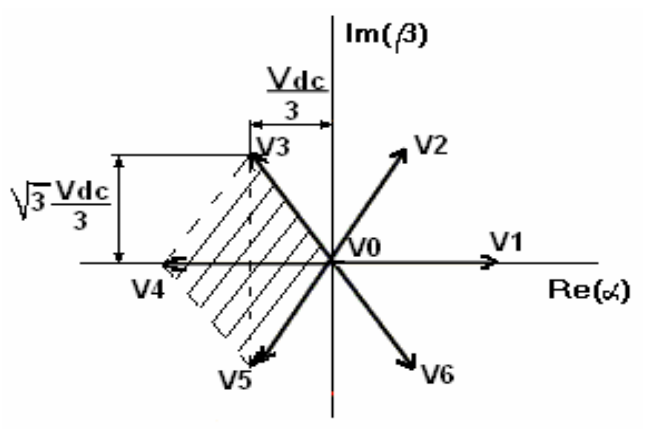

Fig.2 Voltage space-vectors in proposed converter

As can be seen from Fig. 3 the dc-phase components of the capacitors forming the voltage space vector $\mathbf{V}_{\text {off }}$ can be derived as

$V_{\mathrm{fa} 0}=-1 / 3 V_{\mathrm{dc}}, V_{\mathrm{fb} 0}=V_{\mathrm{fc} 0}=1 / 6 V_{\mathrm{dc}}$ 


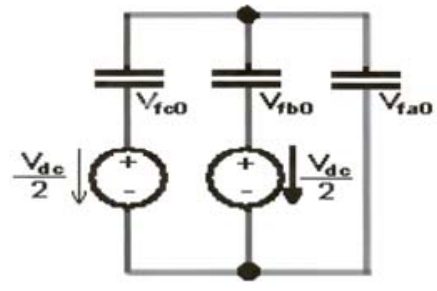

Fig.3 DC-offset on filter capacitors

From Fig.4 (on left) we can see the voltage spacevector diagram for the B4 configuration after subtracting

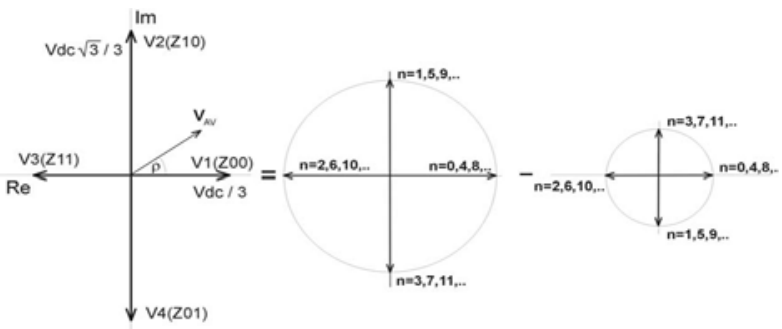

Fig.4 Decomposition of the voltage space-vectors into symmetrical sequences

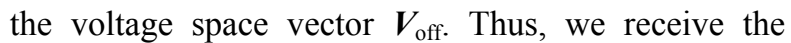
voltage space-vectors as shown in shaded area in Fig.2.

These vectors are $\pi / 2$ away from each other. Additionally, these vectors do not have the same amplitude as in the classical B6 connection. Vectors lying in the real axis have the amplitude of $V_{d c} / 3$, whereas the vectors lying in the imaginary axis have the amplitude of $\sqrt{3} V_{d c} / 3$. The vectors in Fig.4 have the following denotation

$\boldsymbol{V}_{1}(\mathrm{Z} 00), \boldsymbol{V}_{2}(\mathrm{Z} 10), \boldsymbol{V}_{3}(\mathrm{Z} 11), \boldsymbol{V}_{4}(\mathrm{Z} 01)$

where $\left(Z, S_{\mathrm{B}}, S_{\mathrm{C}}\right)$ in denotes the inverter switching states, in which $S_{\mathrm{i}}=1(i=B, C)$ if the upper leg switch is on and $S_{\mathrm{i}}=0$ if the upper leg switch is off. $Z$ means zero pole voltage $v_{\mathrm{a}}=0$, with permanently connected phase a to a negative de terminal $(Z \rightarrow 0)$.

To preserve four-switch symmetry we can decompensate the unsymmetrical voltage vector sequence (on left) into the two symmetrical ones, the positive sequence (middle) and negative sequence (right).For the each of the symmetrical sequences we can use the mixed $\mathrm{p}-\mathrm{z}$ approach for an analytical calculation of the source $\boldsymbol{i}_{\mathrm{S}}$, filter $\boldsymbol{i}_{\mathrm{F}}$ and load currents $i_{\mathrm{L}}$.
We can write in Fig.1 for space-vectors notation

$\boldsymbol{v}_{\mathrm{S}}=\left(R_{\mathrm{S}}+p L_{\mathrm{S}}\right) \boldsymbol{i}_{\mathrm{S}}+\left(R_{\mathrm{F}}+p L_{\mathrm{F}}+1 /\left(p C_{\mathrm{F}}\right)\right) \boldsymbol{i}_{\mathrm{F}}+\boldsymbol{v}^{*}$

$\boldsymbol{i}_{\mathrm{S}}=\boldsymbol{i}_{\mathrm{L}}+\boldsymbol{i}_{\mathrm{F}}$

where $\boldsymbol{v}^{*}$ is the output PWM converter voltage against point " $n$ " of the ac supply. This voltage can be decomposed into the two symmetrical sequences: positive $\boldsymbol{V}_{P}(n, \varepsilon)$ and negative $\boldsymbol{V}_{N}(n, \varepsilon)$

$$
\begin{aligned}
& \boldsymbol{v}^{*}(n, \varepsilon)=\left|\boldsymbol{V}_{A V}\right| \boldsymbol{e}^{j \rho}=\boldsymbol{V}_{P}(n, \varepsilon)+\boldsymbol{V}_{N}(n, \varepsilon)= \\
& \sum_{k=1}^{3 N_{1}} \sum_{x=1}^{2} V_{d c}(1+\sqrt{3}) \frac{1}{6} f(\varepsilon, k, x) e^{j n \pi} e^{j \frac{\pi}{2} \alpha(k)} e^{j \pi \frac{(x-1)}{2}} \\
& +\sum_{k=1}^{3 N_{1}} \sum_{x=1}^{2} V_{d c}(1-\sqrt{3}) \frac{1}{6} f(\varepsilon, k, x) e^{-j n \pi} e^{-j \frac{\pi}{2} \alpha(k)} e^{-j \pi \frac{(x-1)}{2}}
\end{aligned}
$$

$f(\varepsilon, k, x)$ in (14) is a switching function for the proposed space-vector modulation. Time is expressed in per unit as

$$
t=(n+\varepsilon) T=(n+\varepsilon) \frac{\omega}{2 \pi}
$$

where $n$ is a positive integer (number of the sector) and $0 \leq \varepsilon \leq 1$ is a per-unit time inside of a sector Equations (4) - (6) form the base for the control spacevector algorithm and also for the closed-form solution of the line currents $\boldsymbol{i}_{\mathrm{S}}$. The supply voltage $\boldsymbol{v}_{\mathrm{S}}$ may contain both the positive and negative voltage sequences as

$v_{\mathrm{S}}=v_{\mathrm{SP}}+v_{\mathrm{SN}}$

From (4)-(6) we calculate for given $\boldsymbol{i}_{\mathrm{S}}, \boldsymbol{i}_{\boldsymbol{L}}, \boldsymbol{v}_{\mathrm{S}}$ and the parameters $R_{\mathrm{S}}, L_{\mathrm{S}}, R_{\mathrm{F}}, L_{\mathrm{F}}$ and $C_{\mathrm{F}}$ the control filter reference voltage amplitude $\quad\left|\boldsymbol{V}_{A V}\right|$ and phase $\rho$. These values are used for calculation of the switching times in Space-Vector PWM (SVPWM).

Using the mixed p-z approach [4], we can derive the closed-form analytical expression for the line or the filter currents. As an example we can see in Fig.5a the trajectory of the line current in the complex $\alpha \beta$ plane and its fundamentals harmonics (dotted line). Fig.5a and $5 \mathrm{~b}$ show the real and imaginary part of the line current space vector. The real part of the current spacevector is also current in phase ,a“. Fig.5d shows the non-linear load current in phase ,a“. This load spacevector current was modeled by the following equation (only three load current harmonics were considered in the example)

$$
\boldsymbol{I}_{\mathrm{L}}(\mathrm{n}, \boldsymbol{\varepsilon})=\boldsymbol{I}_{1} \mathrm{e}^{\mathrm{j} \omega(\mathrm{n}+\varepsilon) \mathrm{T}}+\boldsymbol{I}_{1} / 5 \mathrm{e}^{-5 \mathrm{j} \omega(\mathrm{n}+\varepsilon) \mathrm{T}}+\boldsymbol{I}_{1} / 7 \mathrm{e}^{7 \mathrm{j} \omega(\mathrm{n}+\varepsilon) \mathrm{T}}
$$


The following parameters were used for the analytical calculations:

$V_{\mathrm{dc}}=1250 \mathrm{~V}, V_{\mathrm{SP}}=300 \mathrm{~V}, V_{\mathrm{SN}}=15 \mathrm{~V}, \omega=314 \mathrm{~s}^{-1}, R_{\mathrm{S}}=0.1$ $\Omega, R_{\mathrm{F}}=1.0 \Omega, L_{\mathrm{S}}=0.025 \mathrm{H}, L_{\mathrm{F}}=0.005 \mathrm{H}, C_{\mathrm{F}}=0.0001 \mathrm{~F}$ $f_{\mathrm{SW}}=3 \mathrm{kHz}$

a)
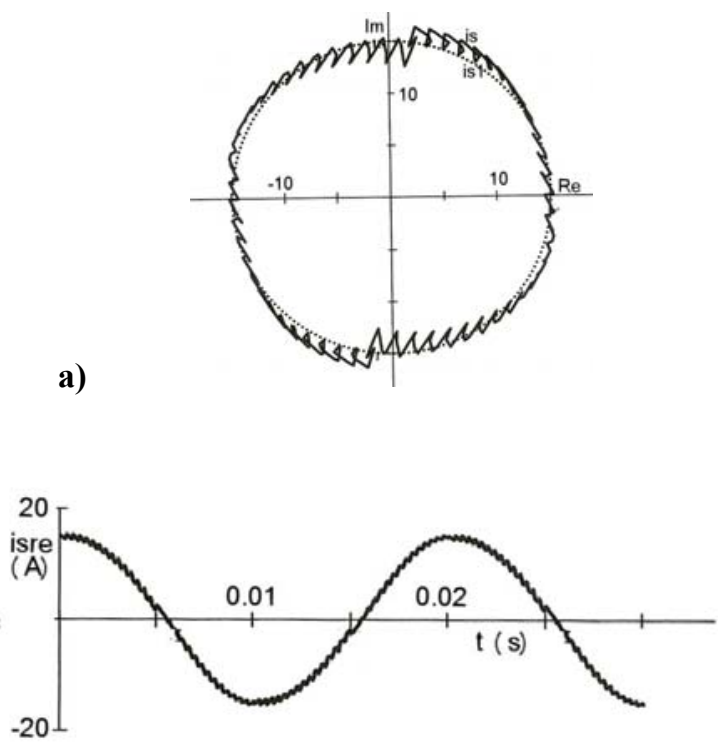

b)

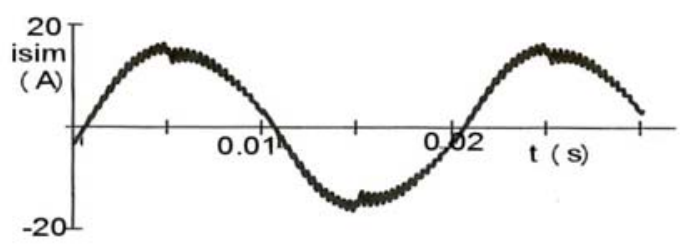

c)

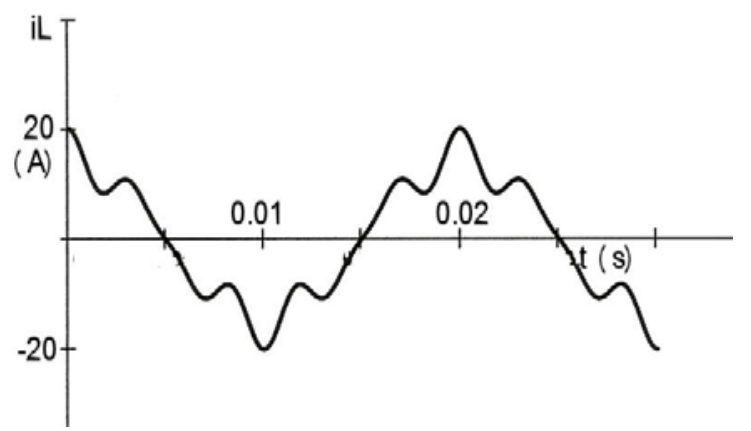

d)

Fig. 5 Analytical results. The line current space $\operatorname{vector}(5 a)$ and its real (5b) and imaginary $(5 c)$ parts. The phase load current (5d)

\section{Experiments}

We have also verified the proposed method through experimentation. The experimental system consists of a three-phase inverter $R_{\mathrm{f}}, L_{\mathrm{f}}$, and $C_{\mathrm{f}}$ passive filter and nonlinear load ( three-phase uncontrolled rectifier). The setup contains also digital control unit. The inverter is

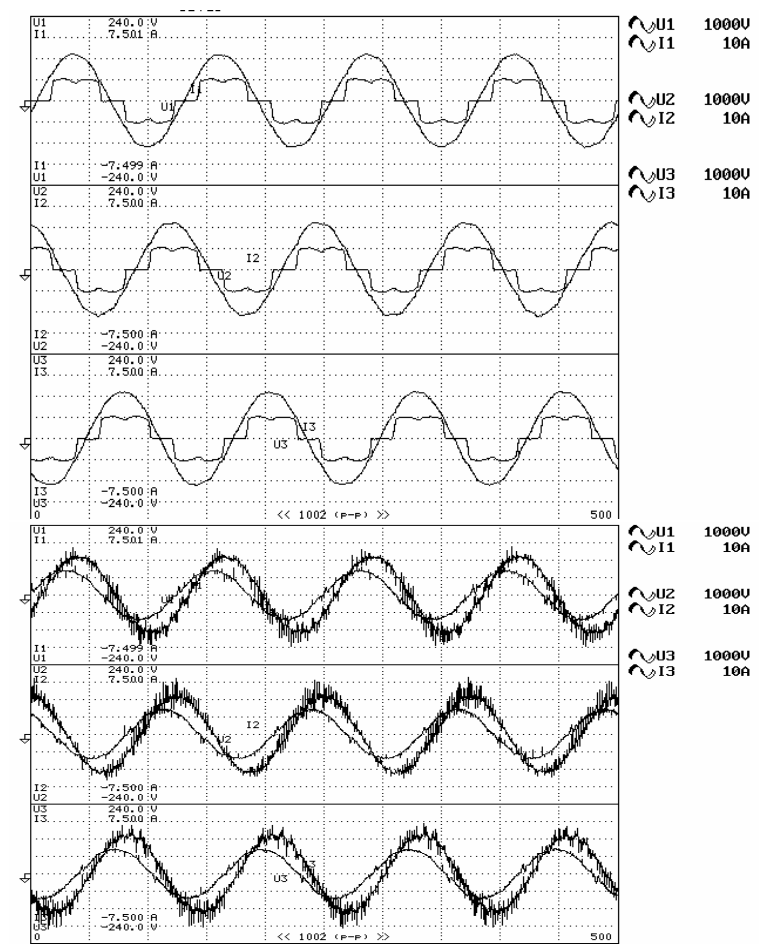

Fig.6 Experimental results. Voltages (60V/div), load currents (upper traces), and line currents (bottom space)(1.875 A/div)

an integrated power module MITSUBISHI PS12038 (1200 V, $25 \mathrm{~A}, 15 \mathrm{kHz}$ at maximum). The digital control unit, based on the dSPACE control system DS 1103, generates firing signals for the PWM rectifier. In Fig. 6 we can see the phase load currents and also line load currents together with the phase voltages. The parameters of the experimental setup are as follows:

$V_{\mathrm{dc}}=286 \mathrm{~V}, V_{\mathrm{SP}}=300 \mathrm{~V}, \omega=314 \mathrm{~s}^{-1}$,

$R_{\mathrm{S}}=1.4 \Omega, \quad R_{\mathrm{F}}=1.0 \Omega, L_{\mathrm{S}}=0.0111 \mathrm{H}, L_{\mathrm{F}}=0.005 \mathrm{H}$, $C_{\mathrm{F}}=0.000041 \mathrm{~F}, \mathrm{C}_{\mathrm{dc}}=0.000832 \mathrm{~F}, f_{\mathrm{SW}}=4 \mathrm{kHz}$

As can be seen the phase line currents are controlled to be sinusoidal. The load phase currents are highly distorted as they supply nonlinear load consisting of six-phase rectifier with $R, C$ load. 


\section{Conclusion}

A three-phase four-switch converter for the hybrid filter with the new four-switch converter topology is implemented and analytically investigated. The scheme with a reduced number of powers switches, improving the filter efficiency. Based on the control algorithm, sinusoidal line currents compensating the non-linear load currents are achieved by the adopted converter. Finally, an original closed-form solution of steady-state line currents, based on the mixed $\mathrm{p}-\mathrm{z}$ approach is introduced. The analytical results and experiment tests on the component minimized three-phase PWM HPF are shown as well to demonstrate the validity and effectiveness of the proposed control scheme.

\section{References}

[1] Akagi, H.: "New Trends in Active Filters for Power Conditioning," IEEE Transactions on IA-32, No. 6, 1996, 1312-1322.

[2] Peng, F. Z.: "Harmonic Sources and Filtering Approaches," IEEE IA Magazine, July/August $2001,18-25$

[3] Skramlík, J., Valouch, V.: "Coupled Feedforward and Feedback Control Strategy of Parallel Hybrid Filter for Harmonic Mitigation, "Inter. Conference on Renewable Energies and Power Quality ICREPQ 05, Zaragoza, Spain, March 16-18, pp. $1-3,2005$.

[4] Klima, J: “Mixed p-z approach for time-domain analysis of voltage source inverters with periodic pulsewidth modulation." IEEE Transactions on Circuits and Systems II. 2004, No.10, October, pp.529- 536.
[5] Lin,B.,R and Wei, T.,C“ Analysis and implementation of a three-phase two-leg neutral point clamped converter based on space vector PWM for power factor correction. IEE Proceedings-Electric Power Applications, Vol.151, No1, pp.38-46, Jan. 2004.

[6] Klima, J. "Analytical closed-form solution of a space-vector modulated VSI feeding an induction motor drive," IEEE Transaction on Energy Corversion, vol.17, no2,pp.191-196.June 2002.

[7] Klima, J., Skramlik, J., and Valouch,V.: "An Analytical Modelling of Three-Phase Four-Switch PWM Rectifier Under Unbalanced Supply Conditions," IEEE Trans. on Circuits and System II, 2007, No.6, pp. 1155-1159.

\section{Acknowledgement}

The financial supports of the Academy of Sciences of the Czech Republic (Institutional Research Plan Z20570509 of the Institute of Thermomechanics) and of the Grant Agency of the Academy of Sciences of the Czech Republic (Project No. IAA200760703) are highly acknowledged. 